\title{
Oxygen matters: tissue culture oxygen levels affect mitochondrial function and structure as well as responses to HIV viroproteins
}

\author{
This article has been corrected since Online Publication and a corrigendum has also been published
}

\author{
LM Tiede ${ }^{1,2}$, EA Cook ${ }^{1}$, B Morsey $^{1}$ and HS Fox ${ }^{*, 1,2}$
}

Mitochondrial dysfunction is implicated in a majority of neurodegenerative disorders and much study of neurodegenerative disease is done on cultured neurons. In traditional tissue culture, the oxygen level that cells experience is dramatically higher (21\%) than in vivo conditions (1-11\%). These differences can alter experimental results, especially, pertaining to mitochondria and oxidative metabolism. Our results show that primary neurons cultured at physiological oxygen levels found in the brain showed higher polarization, lower rates of ROS production, larger mitochondrial networks, greater cytoplasmic fractions of mitochondria and larger mitochondrial perimeters than those cultured at higher oxygen levels. Although neurons cultured in either physiological oxygen or atmospheric oxygen exhibit significant increases in mitochondrial reactive oxygen species (ROS) production when treated with the human immunodeficiency virus (HIV) virotoxin trans-activator of transcription, mitochondria of neurons cultured at physiological oxygen underwent depolarization with dramatically increased cell death, whereas those cultured at atmospheric oxygen became hyperpolarized with no increase in cell death. Studies with a second HIV virotoxin, negative regulation factor (Nef), revealed that Nef treatment also increased mitochondrial ROS production for both the oxygen conditions, but resulted in mitochondrial depolarization and increased death only in neurons cultured in physiological oxygen. These results indicate a role for oxidative metabolism in a mechanism of neurotoxicity during HIV infection and demonstrate the importance of choosing the correct, physiological, culture oxygen in mitochondrial studies performed in neurons.

Cell Death and Disease (2011) 2, e246; doi:10.1038/cddis.2011.128; published online 22 December 2011

Subject Category: Neuroscience

Mitochondria are implicated in a variety of neurodegenerative conditions including aging, Parkinson's disease, Huntington's disease, multiple sclerosis and Alzheimer's disease. A prime function of mitochondria is to reduce oxygen to water during oxidative phosphorylation to produce adenosine tri-phosphate (ATP). Altered levels of oxygen are detected by the mitochondrion and communicated to the cell as a whole via reactive oxygen species (ROS). ${ }^{1,2}$ The vast majority of tissue culture is currently conducted in incubators with an oxygen tension matching that of atmospheric oxygen levels (21\%). However, this is much greater than the oxygen levels experienced in vivo. Although exposure to lower physiological oxygen conditions $(2-5 \%)$ has been documented to increase survival, proliferation and dopaminergic differentiation of cultured neurons, ${ }^{3-5}$ most experiments continue to be done predominantly at atmospheric oxygen levels. This transcends the nervous system as it has been found that immune cells, when cultured at physiological oxygen, exhibit markedly altered responses to stimuli. ${ }^{6,7}$

One disconcerting phenomenon associated with persistent human immunodeficiency virus (HIV) infection is neurode- generation. Though HIV-associated dementia and encephalitis have decreased with successful treatment of HIV by combination antiretroviral therapy, cognitive deficits still occur within otherwise healthy HIV-infected individuals ${ }^{8,9}$ have been revealed. Prime candidates for inducing neuronal dysfunction are viral proteins, as production of early viral products can persist even in the presence of therapy and absence of productive viral replication.

One such protein, the trans-activator of transcription (Tat), activates transcription of the viral genome with other welldocumented functions, and has been shown to be neurotoxic ${ }^{10-18}$ and cause both mitochondrial hyperpolarization and depolarization. ${ }^{19-23}$ Interestingly, examination in immune cells studied under various tissue culture oxygen levels revealed that Tat was toxic to lymphocytes cultured in atmospheric oxygen, but stimulated cells cultured at physiologically relevant oxygen. ${ }^{24}$

Another viral protein, the 'negative regulation factor' (Nef), can be transferred to uninfected cells ${ }^{25-28}$ and released into the extracellular environment. ${ }^{29,30}$ Nef has also been shown

\footnotetext{
${ }^{1}$ Department of Pharmacology and Experimental Neuroscience, University of Nebraska Medical Center, Omaha, NE, USA and ${ }^{2}$ Nebraska Center for Virology, University of Nebraska Lincoln, Lincoln, NE, USA

${ }^{*}$ Corresponding author: HS Fox, Department of Pharmacology and Experimental Neuroscience, University of Nebraska Medical Center, 985800 Nebraska Medical Center, Omaha, NE 68198-5800, USA. Tel: + 402559 4821; Fax: + 402559 7495; E-mail: hfox@ unmc.edu

Keywords: mitochondria; culture oxygen; neurodegeneration; neurons; HIV

Abbreviations: ROS, Reactive oxygen species; HIV, human immunodeficiency virus; Tat, trans-activator of transcription; Nef, negative regulation factor; Dhr123, dyhydrorhodamine 123; ATP, adenosine tri-phosphate; ADP, adenosine di-phosphate; RSNs, rat striatal neurons; Mg, magnesium

Received 16.5.11; revised 14.11.11; accepted 14.11.11; Edited by A Finazzi-Agró
} 
to be toxic to a variety of cell types in brain, ${ }^{31-33}$ and found to increase ROS production, depolarize mitochondria, and increase activated caspase production in astrocytes cultured under hypoglycemic conditions. ${ }^{34}$

Here, we investigated the influence of tissue culture oxygen levels in neurotoxicity of HIV viroproteins in primary rat striatal neurons (RSNs). Although levels of ROS production induced by Tat were similar regardless of oxygen levels, effects of Tat on mitochondrial polarization and neuron survival depended critically on oxygen levels, perhaps contributing to divergent results reported by others and with important ramifications for the mechanism of toxicity. Similarly, Nef treatment increased ROS production regardless of oxygen level, but resulted in mitochondrial depolarization and increased cell death only in neurons cultured under physiological oxygen conditions.

\section{Results}

Mitochondrial polarization changes with tissue culture oxygen levels. Mitochondrial polarization is a critical measure of mitochondrial function and was determined using ratiometric $\mathrm{JC}-1$ imaging. In the presence of high membrane potential, JC-1 forms dimers and the fluorescence shifts from green to red. By assessing the ratio of the red to green fluorescence, mitochondrial potential can be compared between samples with the higher ratio corresponding to a higher mitochondrial membrane potential. The images in Figure 1 clearly indicate that the highest mitochondrial potential in primary RSNs occurred in the $2 \%$ oxygen culture condition. The mitochondrial polarization values ranged from 1.33 under uncoupling conditions
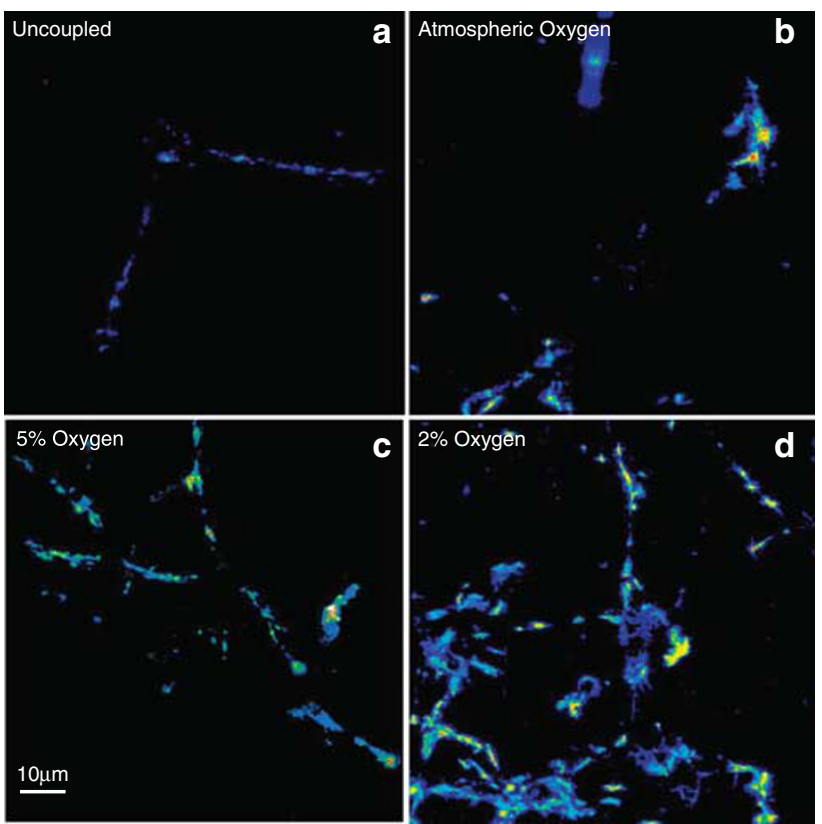

Figure 1 JC-1 polarization ratio images for uncoupled neurons (a) neurons cultured in atmospheric oxygen (b), $5 \%$ oxygen (c) or $2 \%$ oxygen (d) Images were obtained by dividing the red JC-1 fluorescence image by the green JC-1 fluorescence image and using a 16-color lookup table. The black indicates a ratio value of 0 (background) while the white represents the maximum polarization. Scale bar indicates $10 \mu \mathrm{m}$ neurons to 3.8 for neurons cultured in $2 \%$ oxygen. These results can be seen in Figure $2 a$. As such the optimal oxygen level was $2 \%$ as determined by the maximum mitochondrial membrane potential because high mitochondrial potential indicates a greater capacity for ATP production. These results were easily repeatable in three biological replicates.

\section{Mitochondrial ROS production rates vary with tissue} culture oxygen levels. Dihydrorhodamine 123 is a fluorophore that is localized to mitochondria and fluoresces only upon oxidation and as such can be used to make comparative measures of the rate of ROS production. The ROS production rate was greatest for the neurons cultured in $5 \%$ oxygen $(0.18 \mathrm{ADU} / \mathrm{min})$. Lower values were obtained in the atmospheric condition with an ROS production rate determined to be $0.07 \mathrm{ADU} / \mathrm{min}$, and the lowest $(0.002 \mathrm{ADU} / \mathrm{min})$ in the neurons cultured at $2 \%$ oxygen as shown in Figure $2 \mathrm{~b}$. As high levels of ROS are damaging to cells, these results indicate that in terms of minimizing ROS production culturing RSNs at $2 \%$ oxygen is the most favorable.

a

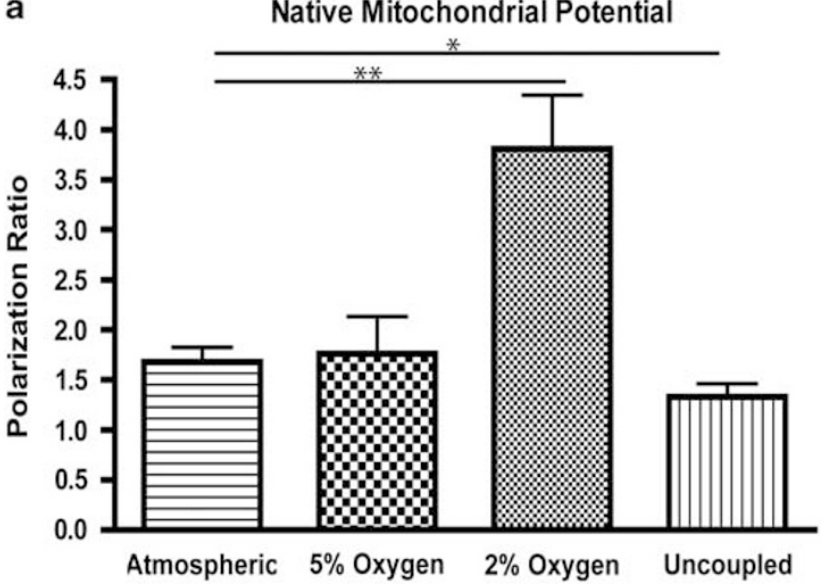

b

\section{Native ROS Production Rate}

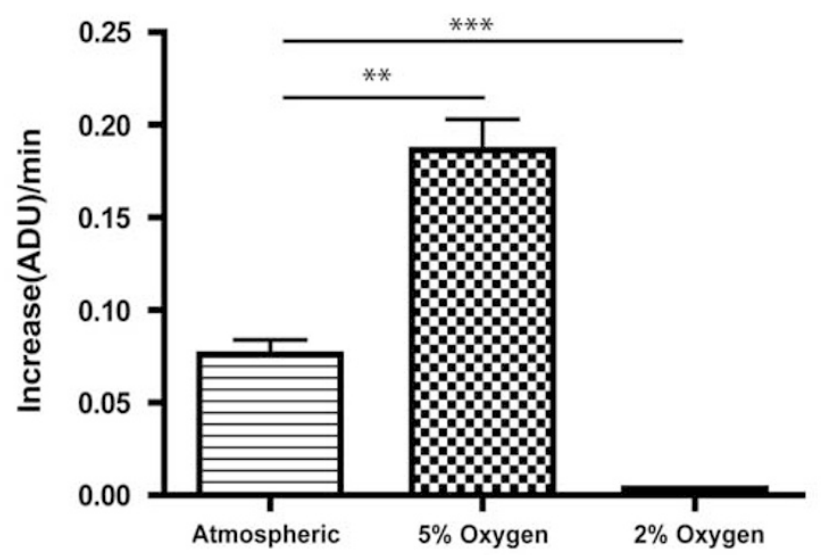

Figure 2 Quantification of effects of culture oxygen on mitochondrial polarization (a) as given by the JC-1 polarization ratio and reactive oxygen species production (b) indicated by the rate of Dhr123 fluorescence increase. ${ }^{*} P<0.05$, ${ }^{\star \star} P<0.01$ and ${ }^{* \star *} P<0.001$ for $N=3$ biological replicates 


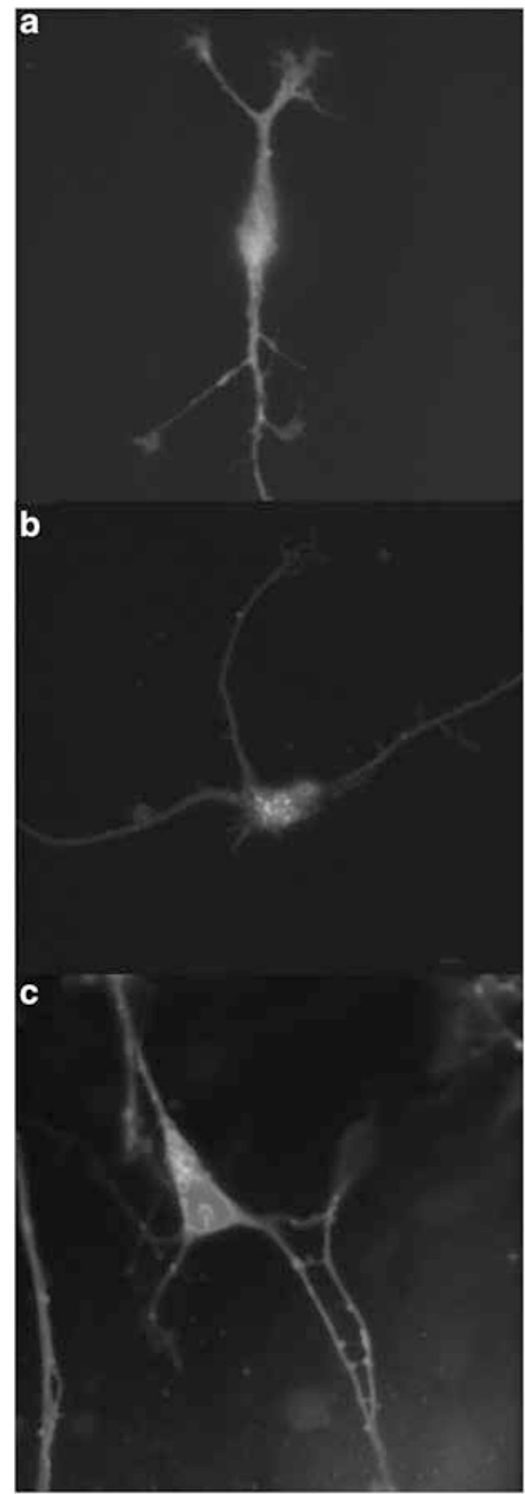

d

Native Mitochondrial Network Size

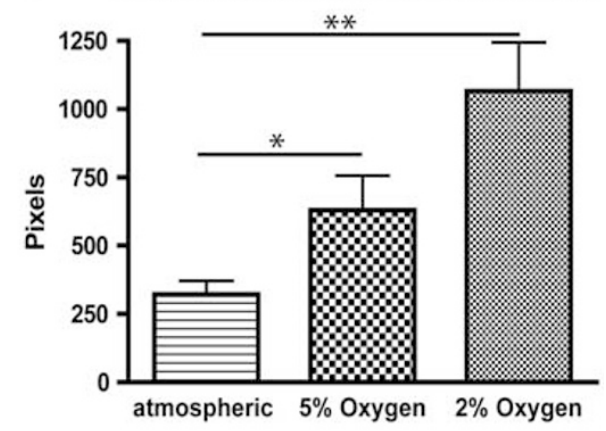

e

\section{Native Mitochondrial Fraction}

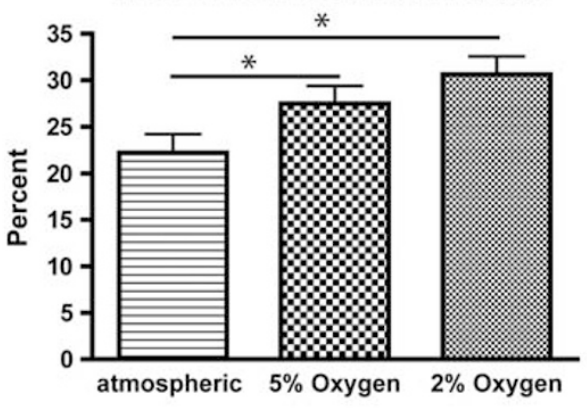

f

Native Mitochondrial Perimeter

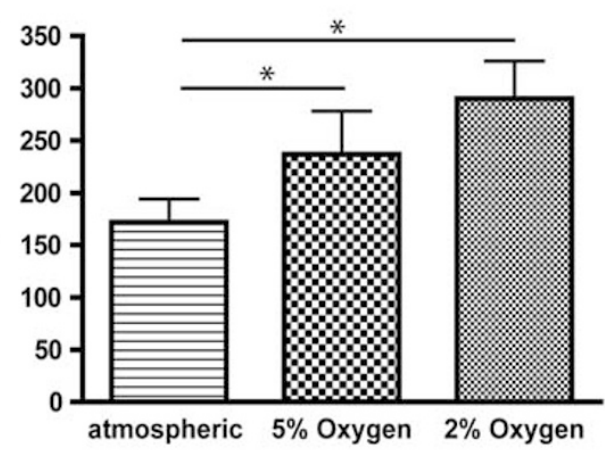

Figure 3 Images of neurons labeled with Mitotracker Red that have been cultured in atmospheric (a) $5 \%$ oxygen (b) and $2 \%$ oxygen (c) as well as quantification of mitochondrial morphology including network size (d), area fraction (e) and perimeter (f) determined from all Mitotracker Red images. ${ }^{\star} P<0.05$ and ${ }^{\star \star} P<0.01$ for $N=3$ biological replicates

Mitochondrial morphology and abundance differ according to tissue culture oxygen levels. Mitotracker Red CM- $\mathrm{H}_{2} \mathrm{XRos}$ was used to label functional mitochondria in neurons to investigate changes in the mitochondrial distribution and morphology (Figure 3). Although many parameters of shape and size could be analyzed, we have chosen to focus on network size, denoting the average size of the extensive mitochondrial networks in the neurons, perimeter, representing the outline of the networks, and fraction, which indicates the proportion of the area of the image occupied by the mitochondria.

The network size is maximal (1066 pixels) in neurons cultured at $2 \%$ oxygen, and decreases to 630 pixels in RSNs cultured at $5 \%$ oxygen and again to 321 pixels in neurons cultured in atmospheric oxygen (Figure $3 \mathrm{~d}$ ). The area fraction and perimeter both follow the same decreasing trend. As shown in Figure $3 e$, the area fraction constituted $30 \%$ in neurons cultured at $2 \%$ oxygen but dropped to $22 \%$ cultured in atmospheric oxygen. For the perimeter (Figure 3f), neurons cultured in $2 \%$ oxygen were maximal at 290 pixels, whereas this dropped to 172 pixels at atmospheric oxygen. These results agree with those seen in transmission electron microscopy images (Figure 4) where at $2 \%$ oxygen the mitochondria were seen as having the greatest length and elongated shapes, whereas those cultured in atmospheric oxygen tended to be shorter in length with a higher proportion of rounded mitochondria.

Mitochondrial polarization changes with Tat treatment. As the neurotoxic effects of Tat are well studied, but mechanistic explanations are quite diverse and sometimes contradictory, we first examined the effects of Tat on mito- 


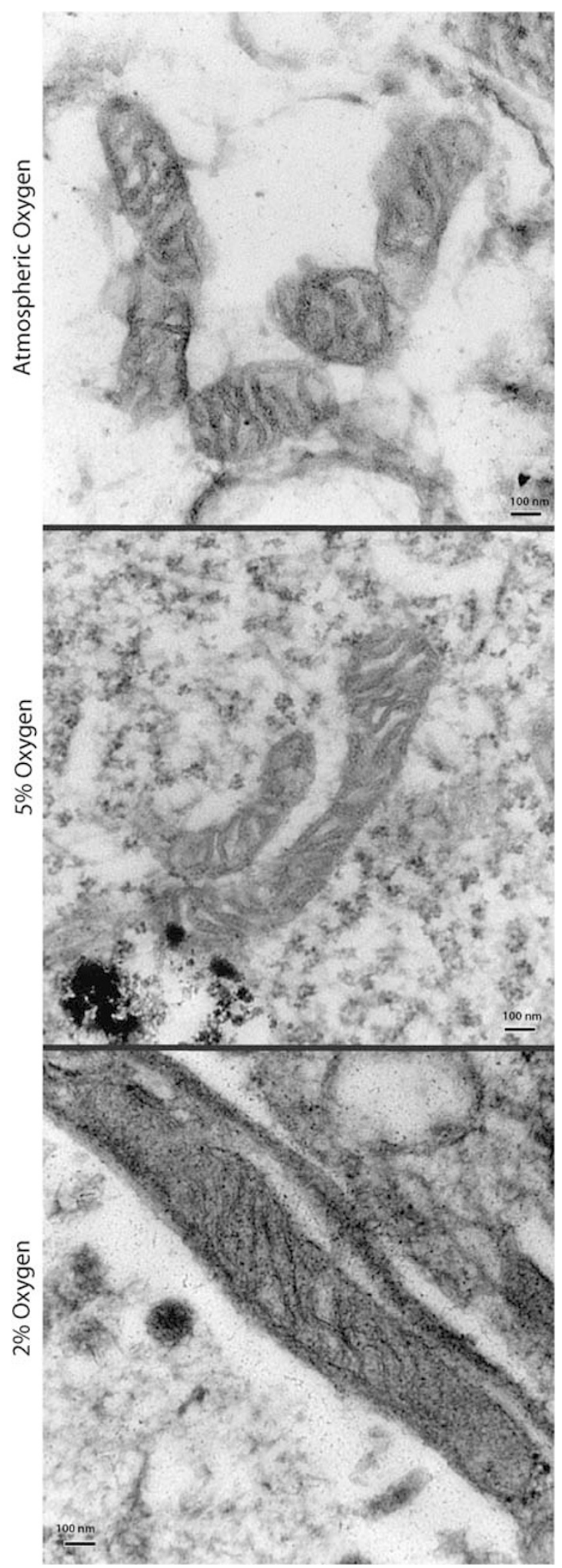

Figure 4 Transmission electron microscopy images of mitochondria in neurons cultured in atmospheric, $5 \%$ and $2 \%$ oxygen. Scale bars indicate $100 \mathrm{~nm}$ a

Tat Mitochondrial Potential

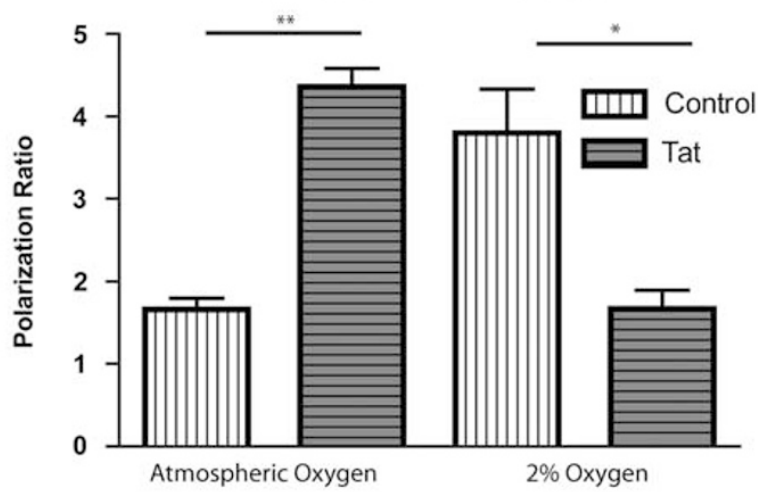

b

Tat ROS Production

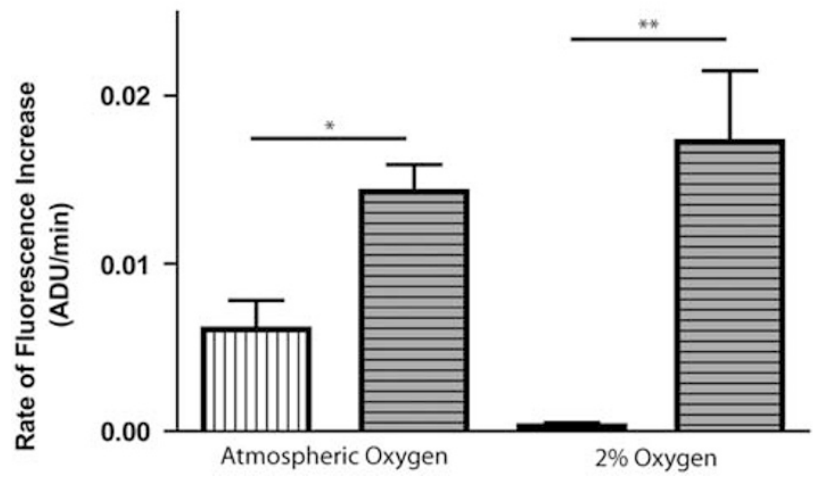

Figure 5 Quantification of effects of Tat on mitochondrial polarization (a) as given by the JC-1 polarization ratio and reactive oxygen species production (b) indicated by the rate of Dhr123 fluorescence increase. ${ }^{*} P<0.05$ and ${ }^{* *} P<0.01$ for $N=3$ biological replicates

chondria from primary neurons cultured under physiological $(2 \%)$ and atmospheric (21\%) oxygen. As above, the baseline polarization ratio of the controls for the untreated $2 \%$ oxygen cultures is significantly higher than that of the atmospheric conditions (Figure 5a).

When treated with Tat $(100 \mathrm{ng} / \mathrm{ml})$, there was a significant $(P<0.01)$ hyperpolarization of mitochondria in neurons cultured in atmospheric oxygen more than doubling the polarization ratio from $1.6 \pm 0.2$ in controls to $3.8 \pm 0.5$ with Tat treatment (Figure 5a). In contrast, neurons cultured in $2 \%$ oxygen level underwent significant $(P<0.05)$ mitochondrial depolarization with Tat treatment, here more than halving the polarization ratio from controls with a $4.3 \pm 0.2$ ratio to $1.6 \pm 0.1$ with Tat treatment (Figure 5a).

ROS production increases following Tat treatment. In Figure $5 \mathrm{~b}$ an increase of ROS is clearly indicated by the heightened rate of fluorescence increase in neuronal cultures treated with $100 \mathrm{ng} / \mathrm{ml}$ Tat for both $2 \%$ oxygen and atmospheric oxygen conditions. The increase is significantly $(P<0.01)$ greater for the case of $2 \%$ oxygen than for the cultures maintained in atmospheric oxygen. However, this result appears to be primarily the result of the lower ROS production rate observed in the $2 \%$ oxygen culture untreated condition compared with the untreated 
atmospheric oxygen cultures, as there is not a statistically significant difference in the rates of ROS production for the Tat-treated cultures.

Thus, our work reveals disparate findings regarding the effects of Tat on neurons. Using parallel cultures under different oxygen conditions, non-physiological atmospheric conditions led to hyperpolarization in response to Tat, whereas with physiological oxygen depolarization occurred. In both the oxygen conditions ROS production increased, however, the magnitude of the changes is different for the two cases examined here.

Mitochondrial polarization changes with Nef treatment under different oxygen conditions. Having demonstrated our ability to find different cellular responses to the virotoxin Tat depending on physiological or non-physiological oxygen conditions, we then turned our attention to Nef. Although Nef is less well studied for its neurotoxic properties, the effects of Nef on infected and uninfected cells has gained significant importance with the recent demonstration of the mechanisms of efficient Nef secretion from infected cells and uptake by uninfected cells and its intracellular transfer from infected to uninfected cells. These both lead to striking in vitro and in vivo effects on a number of cell types and physiological systems. ${ }^{26-30}$

We first treated cultures with Nef as well as heat-inactivated Nef as a control. In neuronal cultures maintained in atmospheric oxygen cultures treatment with $100 \mathrm{ng} / \mathrm{ml}$ Nef (Figures $6 \mathrm{a}, \mathrm{b}$ and $7 \mathrm{a}$ ) did not change polarization ratios. In contrast, in cultures maintained at physiological $2 \%$ oxygen, the mitochondrial polarization ratio was significantly $(P<0.0001)$ decreased for the Nef-treated neurons (Figure 6c) compared with the heat-inactivated Nef-treated neurons (Figure 6d) as can be seen in Figure 7a. The control polarization ratio was $3.8 \pm 0.6$, dropping to $0.99 \pm 0.06$ with active Nef.

Mitochondrial ROS production increases with Nef treatment. Similar to the results seen with Tat treatment, the rate of mitochondrial ROS production increased upon treatment with Nef for neurons cultured in both $2 \%$ oxygen and atmospheric oxygen (Figure 7b; Supplementary Figure 1) when compared with their heat-inactivated Nef controls. The ROS production rates did not vary significantly between the Nef-treated cultures of either oxygen condition, though the basal rates were an order of magnitude lower $(0.0004 \pm$ $0.0002 \mathrm{arb} / \mathrm{min}$ ) for the $2 \%$ oxygen controls than for the atmospheric $(0.007 \pm 0.002 \mathrm{arb} / \mathrm{min})$, indicating a greater increase in ROS production for cells cultured at $2 \%$ oxygen when treated with Nef.

Both Tat and Nef decrease ATP levels in neurons cultured at physiological oxygen concentrations. As we found difference with both Tat and Nef effects on mitochondria due to oxygen conditions, we next assessed their effects on the primary function of mitochondria, production of ATP. Magnesium (Mg) Green fluoresces when bound to $\mathrm{Mg}^{2+}$. As ATP binds $\mathrm{Mg}^{2+}$ more strongly than adenosine di-phosphate (ADP), Mg Green imaging can be used to infer changes in the ATP/ADP ratio over time. As shown in, the normalized ratio of $\mathrm{Mg}$ Green fluorescent rates was vastly greater in the $2 \%$ oxygen cultures following

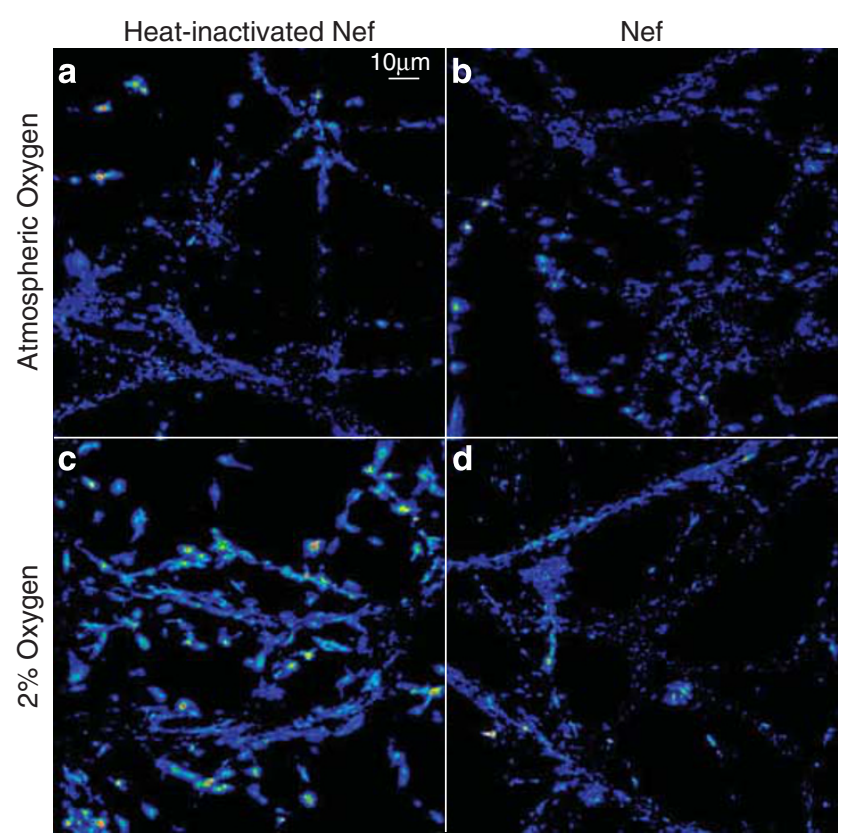

Figure 6 JC-1 polarization ratio images for neurons cultured in atmospheric oxygen ( $\mathbf{a}$ and $\mathbf{b}$ ) or $2 \%$ oxygen ( $\mathbf{c}$ and $\mathbf{d}$ ) following treatment with Nef (b and $\mathbf{d}$ ) or heat-inactivated Nef (a and $\mathbf{c}$ ). Images were obtained by dividing the red JC-1 fluorescence image by the green JC-1 fluorescence image and using a 16-color lookup table. The black indicates a ratio value of 0 (background) while the white represents the maximum polarization. Scale bar indicates $10 \mu \mathrm{m}$

a

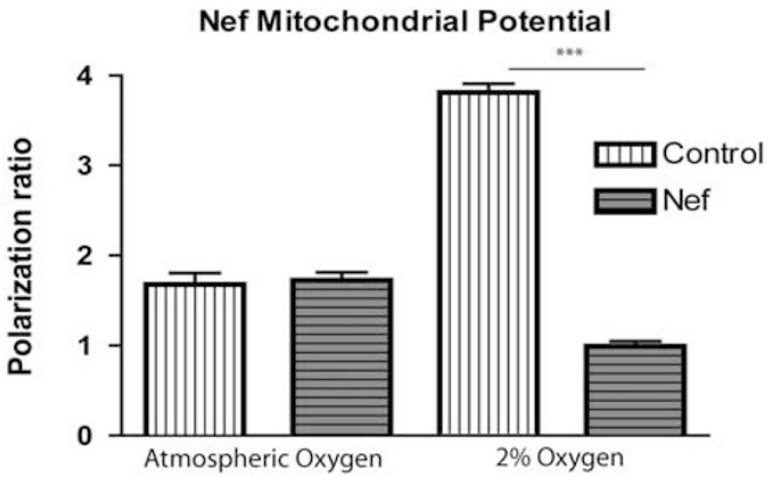

b Nef ROS Production

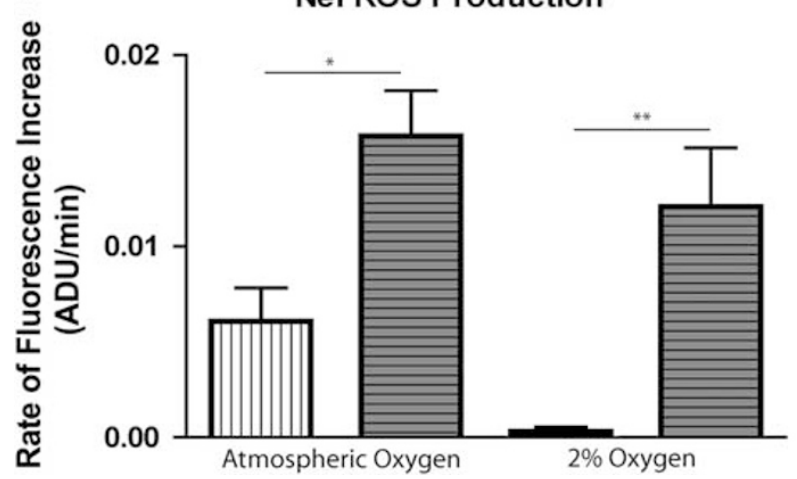

Figure 7 Quantification of effects of Nef on mitochondrial polarization (a) as given by the JC1 polarization ratio and reactive oxygen species production (b) indicated by the rate of Dhr123 fluorescence increase. ${ }^{*} P<0.05$ and ${ }^{* *} P<0.01$ and ${ }^{* \star \star} P<0.001$ for $N=3$ biological replicates 
Tat or Nef treatment than in the atmospheric cultures (Figure 8a). As ATP binds Mg more efficiently than ADP, the increased free $\mathrm{Mg}$ suggests the possibility of more dramatically decreased ATP production in the neurons cultured in $2 \%$ oxygen condition upon exposure to Tat or Nef.

Using the luciferase assay as a direct measure of the ATP concentration, we determined that there was a significant $(P<0.01)$ increase in the amount of ATP from $191 \pm 3 \mathrm{nmol} /$ 10000 cells to $253 \pm 16 \mathrm{nmol} / 10000$ cells following $8 \mathrm{~h}$ of treatment with $100 \mathrm{ng} / \mathrm{ml} \mathrm{Nef}$ in atmospheric oxygen (Figure $8 \mathrm{~b}$ ) and no significant change upon treatment with Tat. For the neurons cultured in $2 \%$ oxygen, however, the concentration of ATP significantly $(P<0.05)$ decreased from $200 \pm 6 \mathrm{nM} / 10000$ cells to $184 \pm 2 \mathrm{nM} / 10000$ cells with an $8-\mathrm{h}$ treatment with $100 \mathrm{ng} / \mathrm{ml}$ Nef. Strikingly, there was a dramatic change found upon treatment of neurons cultured in $2 \%$ oxygen with $100 \mathrm{ng} / \mathrm{ml}$ Tat where ATP concentration dropped to $168 \pm 6 \mathrm{nmol} / 10000$ cells following treatment with Tat.

a Free Magnesium Concentration
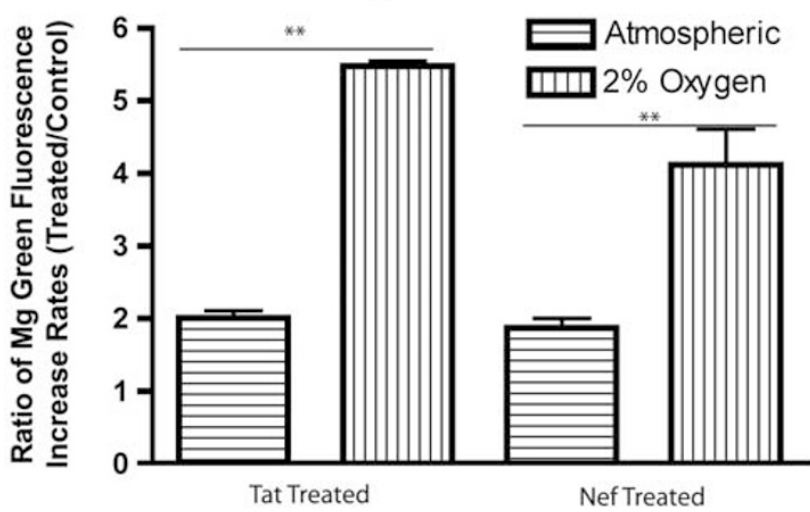

b

Luciferase Assay

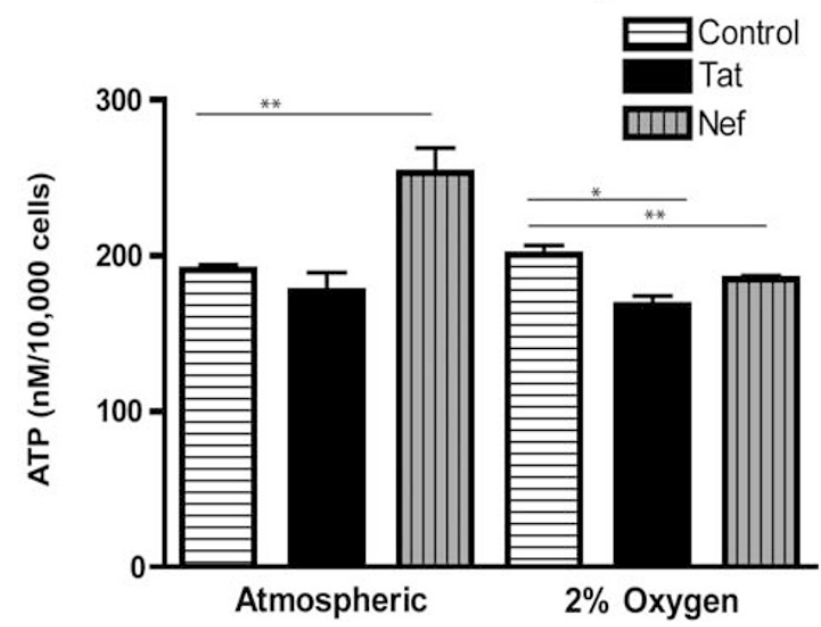

Figure 8 Changes in ATP following Tat or Nef treatment were assessed in two different ways: using magnesium green to measure the changes in free magnesium concentration (a) that can indicate changes in the ADP/ATP ratio, and the luciferase assay (b) that directly measures the amount of ATP present in a sample. ${ }^{*} P<0.05$ and ${ }^{* \star} P<0.01$ for $N=3$ biological replicates
Increased cell death observed in neurons treated with Tat and Nef cultured at physiological oxygen. Calcein/ propidium iodide $(\mathrm{PI})$ staining is a valuable tool used to determine the viability of cultured cells. Calcein is taken up by the cells but is fluorescent only when acted on by esterases and indicates enzyme activity considered to be a marker of healthy cells. $\mathrm{PI}$ is a membrane-impermeable dye that labels DNA, and as such cells exhibiting PI fluorescence have lost membrane integrity and are considered to be dead or dying. Neurons cultured in atmospheric oxygen and treated with Nef exhibited a significant $(P<0.01)$ increase in cell death only when treated with the highest level $(100 \mathrm{ng} / \mathrm{ml})$ of Nef compared with heat-inactivated controls (Figure 9a), and none at all upon treatment with any concentration of Tat. However, neurons cultured in physiological $2 \%$ oxygen exhibited a dose-dependent response to Tat and Nef even in cultures treated with as little as $25 \mathrm{ng} / \mathrm{ml}$ of Tat or Nef (Figure 9b). a

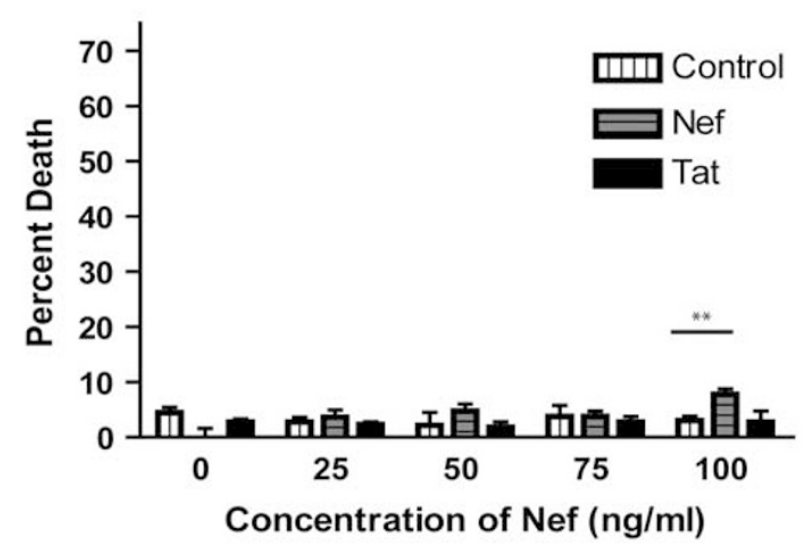

b

$2 \%$ Oxygen

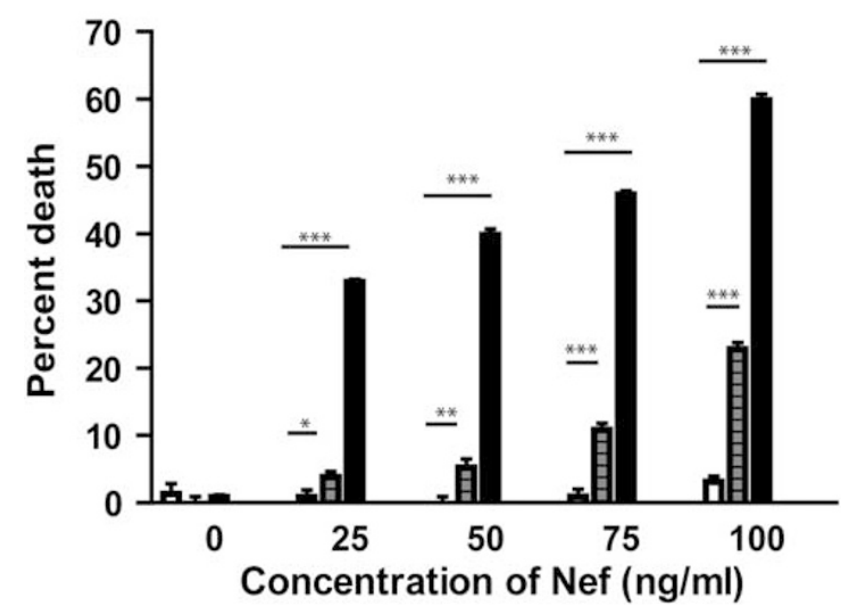

Figure 9 Comparison of the percent of cell death indicated by Calcein and PI staining of neurons cultured with atmospheric oxygen (a) or $2 \%$ oxygen (b) when treated with Tat, Nef or heat-inactivated Nef. ${ }^{\star} P<0.05$ and ${ }^{* \star} P<0.01$ and ${ }^{\star \star \star} P<0.0001$ for a minimum of $N=3$ biological replicates 


\section{Discussion}

It is clear from our results that the unchallenged state of the mitochondria in primary neurons differs depending on the oxygen conditions during cell culture. This is not unreasonable given the delicate balance that must be maintained in order to maximize energy production and minimize the damage from ROS in mitochondria. Our results indicate that there is a state in which maximal mitochondrial function is achieved with minimal ROS production, maximal energy capacity and increased mitochondrial network size and the fraction of the cell occupied by mitochondria. As the result is in the range of oxygen levels expected in the striatum of the rat brain, ${ }^{35}$ it seems reasonable to conclude that the optimal mitochondrial conditions correspond most closely to the native state of the mitochondria in vivo.

The use of less than atmospheric oxygen in culture can raise the question of a hypoxic threshold. It is reasonable to expect that there exist different optimal oxygen levels for the cells derived from different sources corresponding to their natural oxygen level in the organism. In our case $2 \%$ oxygen is within the range of oxygen levels expected in the striatum of the rat brain. Although decreased polarization of mitochondria and increased ROS production are typically noted in studies of hypoxia, ${ }^{36,37}$ the opposite was observed in the RSNs cultured in $2 \%$ oxygen. Taken together with our other results make it highly unlikely that the $2 \%$ oxygen culture condition represents a hypoxic case for these particular primary cells.

For Tat, determining the mechanism of toxicity of this HIVassociated protein has generated a large amount of data that has been contradictory at times. Tat has been shown to both hyperpolarize and depolarize mitochondria, ${ }^{19-23}$ and we have clearly shown that this is indeed the case for RSNs that have been cultured in differing oxygen conditions. Although the hyperpolarization result has become more accepted in recent literature, this result may not have physiological relevance as indicated by the depolarization of neurons cultured in $2 \%$ oxygen, which is similar to the neuron's in vivo environment. It is important to note that culture oxygen is not likely the cause of the divergent results reported by others; however, our results clearly indicate that perturbations in the culture conditions can dramatically alter the response of neuron mitochondria to Tat.

Although ROS production rates were increased by Tat treatment in both the cells cultured at $2 \%$ oxygen and atmospheric oxygen, the increase was more dramatic in the cells cultured in $2 \%$ oxygen. Coupled with the loss of ATP production (see Figure 9) resulting from such dramatic loss of mitochondrial potential, the ROS production could thus be extremely detrimental under these more physiological conditions. It is also important to note that the increase in ROS production may be derived from disruptions of oxidative phosphorylation resulting in increased production of ROS rather than an unrelated effect of Tat for both the cases of hyperpolarization and depolarization. Taken together, it appears that neurons are more strongly affected by Tat than previous studies have led us to believe, and that mitochondrial dysfunction may further complicate other processes already noted to be involved in Tat-related toxicity in neurons. However, this mechanism requires that the initial response of neurons to Tat remains the same regardless of the oxygen conditions, which has not been proven at this point.

Owing to the recent burst of finding on the effects of Nef on uninfected cells, ${ }^{18,29}$ the demonstration of the physiological secretion of Nef from infected cells, ${ }^{29,30}$ and the relative paucity of studies on Nef and neurons, ${ }^{38}$ we then focused our studies on Nef. For Nef there was no effect of the protein on the polarization of mitochondria in neurons cultured in atmospheric oxygen, but the decrease of potential in the $2 \%$ oxygen neuron cultures was equal to that of neurons treated with $10 \mu \mathrm{M}$ carbonyl cyanide m-chlorophenylhydrazone (Figure 2) to cause uncoupling of oxidative phosphorylation. This level of mitochondrial uncoupling usually results in gradual cell death if maintained for prolonged periods. It is possible that the observed cell death can be attributed directly to the loss of ATP production that occurred with the dramatic loss of mitochondrial membrane potential experienced by Neftreated cultures maintained in $2 \%$ oxygen levels. Although the mechanism of this cell death was not directly studied, during calcein/PI imaging, condensation of chromatin and evidence of apoptotic bodies were observed (data not shown), indicating that apoptosis is the likely manner of neuron death resulting from Nef treatment.

As we observed little or no cell death in the atmospheric oxygen cultures treated with Nef and Tat compared with the $2 \%$ oxygen cultures while ROS production increased in both culture conditions, it is reasonable to conclude that ROS production is not solely responsible for the Nef-induced toxicity observed in the $2 \%$ oxygen cultures. Rather it is more likely that the increased ROS is more detrimental because of the decreased ATP levels resulting from Nef treatment that is critical in repair and replacement of damaged lipids and proteins. As the neurons cultured in atmospheric oxygen exhibited increased ATP levels, it is likely that these cells had the energy capacity necessary to make needed repairs to damaged proteins and organelles preventing cell death. Therefore, one proposed mechanism for the Nef-induced toxicity is that decreased energy production resulting from mitochondrial depolarization by Nef results in a reduced capacity to repair proteins damaged by increased levels of ROS and results in eventual apoptosis only in the case of neurons cultured in $2 \%$ oxygen (Figure 10).

One caveat that applies to functional imaging studies is that there are potential issues with the dye used. In the case of $\mathrm{JC}-1$ permanent dimerization has sometimes been noted in the literature. Upon uncoupling with FCCP after making initial measurements the $\mathrm{JC}-1$ polarization ratios always returned within error to the levels obtained from administering dye to the neurons after uncoupling. Also, experimental results were duplicated using the dye tetramethylrhodamine, ethyl ester (TMRE), though TMRE provided less reproducibility from sample to sample and week to week. In addition, in the case of dyhydrorhodamine 123 (Dhr123), the accumulation of dye in mitochondria is dependent upon the potential of the mitochondria. Although this can sometimes skew the results, as we obtained the lowest levels of fluorescence with the highest levels of polarization, this does not seem to be an issue in this study. Also as all time courses showed an increase in fluorescence over time, quenching did not appear to be a significant contributor to the results. 


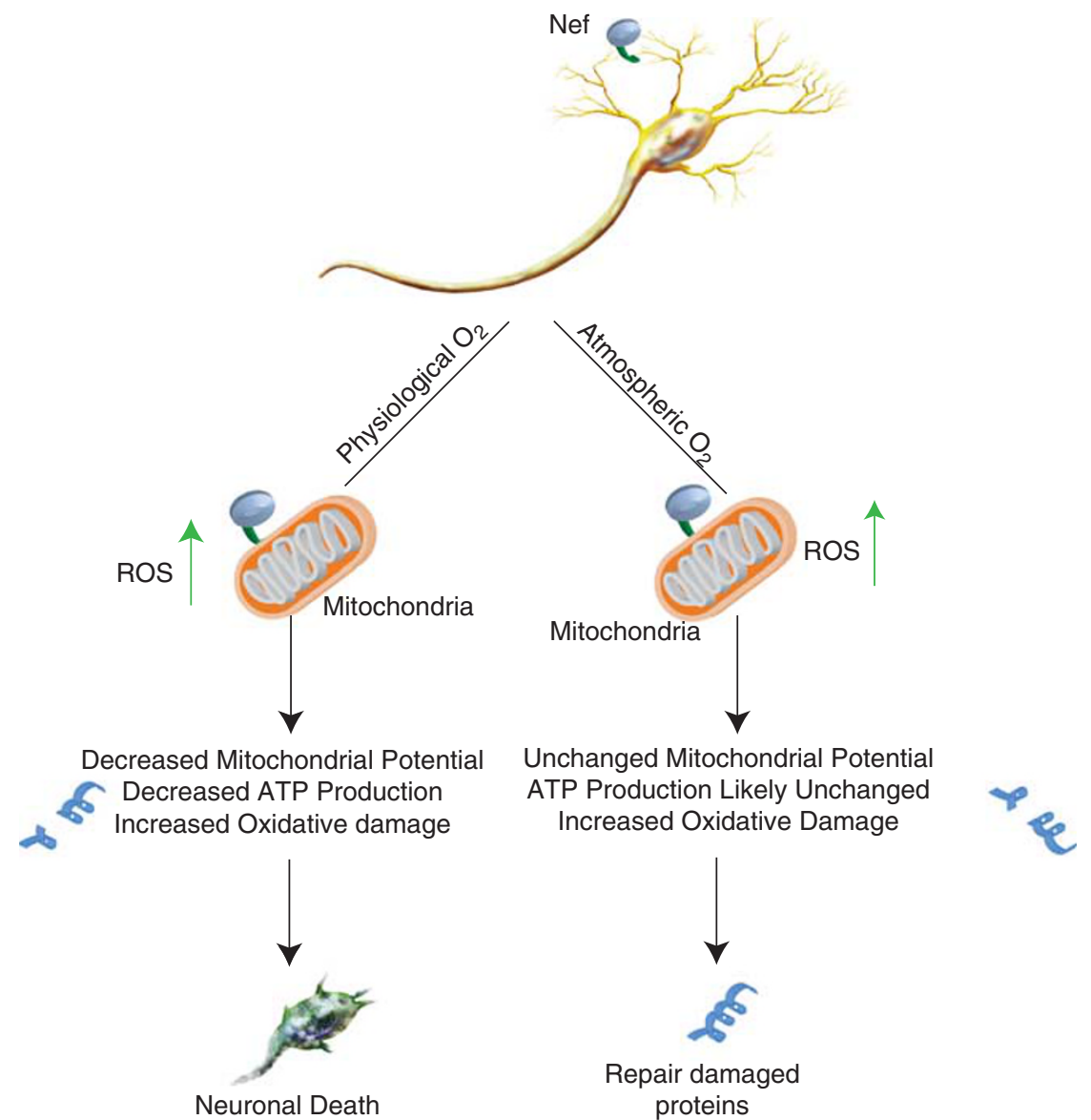

Figure 10 Diagram of proposed mechanism of Nef toxicity for neurons cultured in atmospheric oxygen as well as physiological (2\%) oxygen indicating the pathway to repair the oxidatively damaged proteins or neuronal death, respectively, beginning with alterations in the native mitochondrial structure, function and protein content

It is notable that $\mathrm{Mg}$ Green fluorescence was increased upon treatment with Tat and Nef in both atmospheric and $2 \%$ cultures though ATP levels only dropped significantly in the case of the $2 \%$ cultures. This indicates that there is another source of free Mg resulting from treatment with Tat and Nef that is separate from decreased ATP levels and common to both the culture conditions, correlating with the increased ROS observed in all cultures treated with Tat and Nef.

The road from discovery to application can be extraordinarily complicated in the case of disease and treatment. Things learned in cultured cells have to be confirmed in animal models. Treatments of disease must be translated from the laboratory to the clinic. There are many opportunities along this road for things to break down. In order to minimize the disruptions and failures in attempts to bring successful treatments to the clinic, it is important that the model systems are able to accurately represent the disease state, so that the mechanisms of disease discovered in these models are representative of the natural disease state. Our results show that use of the appropriate oxygen condition reveals not only small effects noted in the atmospheric condition as in the case of Nef, but also that vastly different results can occur as was apparent in the treatment of neurons with Tat. The vastly different mitochondrial response of cultured neurons to Tat could be extremely misleading in the understanding of the process of Tat toxicity, a perfect example of a complication that could limit the translation of knowledge from tissue culture to in vivo cases.

The fact that there are differences in not only the responses of cultured neurons to HIV-associated proteins, but in the natural states of the neurons depending on culture oxygen levels may not be immediately obvious. However, it is important to remember that oxygen, although necessary to neuron survival, is inherently toxic. Furthermore neurons, like most other cells, have the ability to adapt to their environment for survival in culture. Hence, cells cultured in atmospheric oxygen may well be expected to have increased capacity to reduce ROS via increased protein levels of glutathione, superoxide dismutase and other ROS scavengers. The ratios of the different metabolic proteins and their corresponding subunits may also differ in order to regulate energy production under varying oxygen levels. Further experiments are necessary to determine whether this does indeed occur and what relevance it might have to the responses of neurons not only to HIV-associated proteins but also other neuropathic processes.

\section{Materials and Methods}

Neuron harvest and tissue culture. Primary neurons were isolated from rat striatum from E18 embryos (Brain Bits, Springfield, IL, USA) by trituration and centrifugation according to the suggested protocol. Cells were counted and plated in $35 \mathrm{~mm}$, poly-D-lysine-coated dishes (MatTek, Ashland, MA, USA) with No. 1.5 
coverslip buttoms at $5 \times 10^{5}$ cells per dish for imaging or in poly-D-lysine-coated 6 -well plates at $2 \times 10^{5}$ cells per well for western blots. Cells were cultured in Neurobasal MEM supplemented with B-27 and L-glutamate. Our initial studies examined a range of oxygen conditions that are generally considered physiological. After evaluating mitochondrial morphology and function in neurons cultured at atmospheric, 9, 5 and $2 \%$ oxygen, we determined that $9 \%$ oxygen was not significantly different than the atmospheric condition and all of our data here focuses on atmospheric, 5 and $2 \%$ oxygen conditions. For the atmospheric condition, cells were cultured in a standard tissue culture incubator containing $5 \%$ carbon dioxide, whereas for the 2 and $5 \%$ oxygen condition neurons were placed in a chamber (Billups-Rothenberg, Del Mar, CA, USA) charged with a gas mixture containing $2 \%$ oxygen or $5 \%$ oxygen, $5 \%$ carbon dioxide and the remainder nitrogen. Oxygen levels in culture were confirmed using an oxygen electrode. Media were half-exchanged after 5 days and then every 3-4 days thereafter until neurons had been in culture for 14 days. Unless otherwise noted materials were obtained from Sigma-Aldrich (St. Louis, MO, USA).

Imaging. Cells were incubated in the appropriate dye and then imaged on a Nikon swept-field confocal microscope using a $\times 60$ oil-immersion (1.45 NA) objective. A live cell imaging chamber (Pathology Devices, Inc., Westminster, MD, USA) was used to maintain appropriate temperature, oxygen and carbon dioxide conditions during imaging (media oxygenation confirmed using an oxygen electrode). For measuring mitochondrial polarization, cells were incubated in $2 \mathrm{nM} \mathrm{JC}-1$ (Invitrogen, Carlsbad, CA, USA) for 20 min. Images were acquired using a 488-nm excitation, and 536/40-nm and 593/40-nm emission filters for the green monomers and red dimers, respectively. To examine the rate of ROS produced in the mitochondria, neurons were incubated in $10 \mu \mathrm{M}$ Dhr123 (Invitrogen) for $20 \mathrm{~min}$ before imaging. The excitation wavelength used was again $488 \mathrm{~nm}$ with a 536/40-nm fluorescence emission filter. Images were taken at a rate of $1 / \mathrm{min}$ for $10 \mathrm{~min}$ before a new region of interest (ROI) was selected. For mitochondrial morphology experiments cells were incubated in $25 \mathrm{nM}$ Mitotracker Red CM$\mathrm{H}_{2} \mathrm{XRos}$ (Invitrogen) for $20 \mathrm{~min}$. Images were acquired using $568 \mathrm{~nm}$ excitation wavelength and a 593/40-nm emission filter was used to collect the resulting fluorescence.

For calcein and PI staining, cells were incubated in $10 \mu \mathrm{M}$ calcein (Invitrogen) with $10 \mu \mathrm{M} \mathrm{PI}$ (Invitrogen) for $30 \mathrm{~min}$. Cells were imaged on a Zeiss Cell Observer microscope (Carl Zeiss, Thornwood, NY, USA) with appropriate filters to determine whether cells exhibited the presence or absence of calcein and PI staining.

Image analysis. To determine membrane polarization, the ratio of red to green fluorescence of JC-1 images was calculated using $\mathrm{NIH}$ Image J. The outline of individual cells was circled using the freehand tool to create ROls and saved in the multimeasure tool. The average intensity of the same ROls was analyzed in both the red and green channels. Then the ratio was obtained for each region and the average of the ratio was obtained over all ROls for all images. Polarization images were obtained by dividing the red image by the green image and by selecting a 16-color lookup table.

To calculate the rate of ROS production in neurons cultured under various conditions, Dhr123 time series were analyzed in Image J. As previously described, ROls were selected and then propagated through the stack using the multimeasure tool. Average fluorescence values were normalized to the first image ROI. Then all normalized values were averaged at each time point. These average values were plotted and then analyzed with a linear fit to determine the slope. The slope was used to represent the rate of ROS production, and the uncertainty in the slope represented the standard error for the measurement.

In order to investigate changes in mitochondrial morphology, Image $\mathrm{J}$ was used to analyze the Mitotracker red images. The mitochondrial morphology tool was used to select ROls and determine the various parameters used for comparison

For live/dead assays using calcein/PI staining, images were taken, and the number of green and red cells were counted and totaled over all images taken for the experimental condition. The percent of dying cells was determined using the formula: \% death $=\left(N_{\text {red }} / N_{\text {green }}\right) \times 100 \%$.

All statistical analysis was performed using t-tests. All statistical analysis and linear fits were completed using Prism software (GraphPad Software, San Diego, CA, USA).

HIV-associated protein treatments and assays. HIV Tat protein (NIH AIDS Research and Reference Reagent Program, Germantown, MD, USA) was reconstituted in nitrogen gas-deoxygenated water and then diluted in modified
Neurobasal medium to obtain a final concentrations of $25,50,75$ and $100 \mathrm{ng} / \mathrm{ml}$. HIV Nef protein (NIH AIDS Research and Reference Reagent Program) solutions were prepared in the same manner and diluted in media to final concentrations of $25,50,75$ and $100 \mathrm{ng} / \mathrm{ml}$. For imaging studies of mitochondrial potential, structure and ROS production, neurons were incubated in $100 \mathrm{ng} / \mathrm{ml}$ Tat or $100 \mathrm{ng} / \mathrm{ml} \mathrm{Nef}$ for $1 \mathrm{~h}$ before the beginning of imaging. For toxicity curves, neurons were incubated with Neurobasal media containing Tat or Nef for $72 \mathrm{~h}$. Nef that had been heat inactivated at $100{ }^{\circ} \mathrm{C}$ overnight was used as a negative control in all Nef experiments and deoxygenated water alone was used as a control in the experiments involving Tat.

Luciferase assay. Cells were treated for $24 \mathrm{~h}$ with Tat, Nef, or the heatinactivated controls. They were lifted, centrifuged at 7000 r.p.m. and flash frozen before storing at $-80^{\circ} \mathrm{C}$. After thawing, samples were treated and analyzed according to provided protocols for the ATP Determination Kit (Invitrogen).

Fixation for electron microscopy. Neurons were cultured for 14 days according to the conditions above. Media were removed and coverslips were washed three times with PBS before fixation in 3\% paraformaldehyde, $1.5 \%$ glutaraldehyde, $0.1 \mathrm{M}$ sodium cacodylate buffer at a pH of 7.4 , and post fixed in $1 \%$ osmium tetraoxide in $0.10 \mathrm{M}$ sodium cacodylate buffer for $30 \mathrm{~min}$. Afterwards coverslips were dehydrated through an ethanol series and embedded using araldite and polymerized overnight at $65^{\circ} \mathrm{C}$ before sectioning. Thin sections were stained with uranyl acetate and Reynold's lead citrate before examination on a FEI Tecnai G2 transmission electron microscope operated at $80 \mathrm{kV}$.

\section{Conflict of Interest}

The authors declare no conflict of interest.

Acknowledgements. This research was supported by the National Institutes of Health grants MH073490 and MH062261, Nebraska Tobacco Settlement Biomedical Research Development Funds, through a fellowship under Ruth L Kirschstein National Research Service Award 5 T32 Al060547 from the National Institute of Allergy and Infectious Diseases (PI Dr. Charles Wood, Nebraska Center for Virology, University of Nebraska at Lincoln) and National Center for Research Grant Number P20 RR16469 supporting the NE-INBRE Scholars program (PI Dr. James Turpen, UNMC). This is manuscript \#11 from the UNMC Center for Integrative and Translational Neuroscience. We thank Dr. Howard Gendelman for the use of the swept-field confocal microscope and comments on the manuscript. We would also like to thank the Core Electron Microscopy Research Facility at the University of Nebraska Medical Center and Tom Bargar for the use of and expertize with the transmission electron microscopy. The following reagents were obtained through the NIH AIDS Research and Reference Reagent Program, Division of AIDS, NIAID, NIH: HIV-1 Nef (Catalogue no. 11478) and HIV-1 Tat (Catalogue no. 2222, from Dr. John Brady and DAIDS, NIAID).

1. Chandel NS, Maltepe E, Goldwasser E, Mathieu CE, Simon MC, Schumacker PT. Mitochondrial reactive oxygen species trigger hypoxia-induced transcription. Proc Natl Acad Sci USA 1998; 95: 11715-11720

2. Waypa GB, Schumacker PT. Hypoxic pulmonary vasoconstriction: redox events in oxygen sensing. J Appl Physiol 2005; 98: 404-414.

3. Kaplan FS, Brighton CT, Boytim MJ, Selzer ME, Lee V, Spindler K et al. Enhanced survival of rat neonatal cerebral cortical neurons at subatmospheric oxygen tensions in vitro. Brain Res 1986; 384: 199-203.

4. Brewer GJ, Cotman CW. Survival and growth of hippocampal neurons in defined mediums at low density: advantages of a sandwich culture technique or low oxygen. BRES 1989; 494: $65-74$

5. Abbate I, Cappiello G, Longo R, Ursitti A, Spano A, Calcaterra S et al. Cell membrane proteins and quasispecies compartmentalization of CSF and plasma HIV-1 from aids patients with neurological disorders. Infect Genet Evol 2005; 5: 247-253.

6. Atkuri KR, Herzenberg LA, Niemi AK, Cowan T. Importance of culturing primary lymphocytes at physiological oxygen levels. Proc Natl Acad Sci USA 2007; 104: 4547-4552.

7. Atkuri KR, Herzenberg LA. Culturing at atmospheric oxygen levels impacts lymphocyte function. Proc Natl Acad Sci USA 2005; 102: 3756-3759.

8. Ellis R, Langford D, Masliah E. HIV and antiretroviral therapy in the brain: neuronal injury and repair. Nat Rev Neurosci 2007; 8: 33-44.

9. Antinori A, Arendt G, Becker JT, Brew BJ, Byrd DA, Cherner $M$ et al. Updated research nosology for HIV-associated neurocognitive disorders. Neurology 2007; 69: 1789-1799. 
10. Chauhan A, Hahn S, Gartner S, Pardo CA, Netesan SK, McArthur J et al. Molecular programming of endothelin-1 in HIV-infected brain: role of Tat in up-regulation of ET-1 and its inhibition by statins. FASEB J 2007; 21: 777-789.

11. Eugenin EA, King JE, Nath A, Calderon TM, Zukin RS, Bennett MV et al. HIV-tat induces formation of an LRP-PSD-95- NMDAR-nNOS complex that promotes apoptosis in neurons and astrocytes. Proc Natl Acad Sci USA 2007; 104: 3438-3443.

12. Tian C, Erdmann N, Zhao J, Cao Z, Peng H, Zheng J. HIV-infected macrophages mediate neuronal apoptosis through mitochondrial glutaminase. J Neurochem 2008 105: 994-1005.

13. O'Donnell LA, Agrawal A, Jordan-Sciutto KL, Dichter MA, Lynch DR, Kolson DL. Human immunodeficiency virus (HIV)-induced neurotoxicity: roles for the NMDA receptor subtypes. J Neurosci 2006; 26: 981-990.

14. Chen W, Sulcove J, Frank I, Jaffer S, Ozdener H, Kolson DL. Development of a human neuronal cell model for human immunodeficiency virus (HIV)-infected macrophageinduced neurotoxicity: apoptosis induced by HIV type 1 primary isolates and evidence for involvement of the Bcl-2/Bcl-xL-sensitive intrinsic apoptosis pathway. J Virol 2002; 76: 9407-9419.

15. Bonavia R, Bajetto A, Barbero S, Albini A, Noonan DM, Schettini G. HIV-1 Tat causes apoptotic death and calcium homeostasis alterations in rat neurons. Biochem Biophys Res Commun 2001; 288: 301-308.

16. Haughey NJ, Nath A, Mattson MP, Slevin JT, Geiger JD. HIV-1 Tat through phosphorylation of NMDA receptors potentiates glutamate excitotoxicity. J Neurochem 2001; 78: 457-467.

17. Kim HJ, Martemyanov KA, Thayer SA. Human immunodeficiency virus protein Tat induces synapse loss via a reversible process that is distinct from cell death. J Neurosci 2008; 28 12604-12613.

18. Aksenova MV, Aksenov MY, Adams SM, Mactutus CF, Booze RM. Neuronal survival and resistance to HIV-1 Tat toxicity in the primary culture of rat fetal neurons. Exp Neurol 2009; 215: 253-263

19. Norman JP, Perry SW, Kasischke KA, Volsky DJ, Gelbard HA. HIV-1 trans activator of transcription protein elicits mitochondrial hyperpolarization and respiratory deficit, with dysregulation of complex IV and nicotinamide adenine dinucleotide homeostasis in cortical neurons. J Immunol 2007; 178: 869-876.

20. Norman JP, Perry SW, Reynolds HM, Kiebala M, De Mesy Bentley KL, Trejo M et al. HIV-1 Tat activates neuronal ryanodine receptors with rapid induction of the unfolded protein response and mitochondrial hyperpolarization. PLoS One 2008; 3: e3731.

21. Perry SW, Norman JP, Litzburg A, Zhang D, Dewhurst S, Gelbard HA. HIV-1 transactivator of transcription protein induces mitochondrial hyperpolarization and synaptic stress leading to apoptosis. J Immunol 2005; 174: 4333-4344.

22. Kruman II, Nath A, Mattson MP. HIV-1 protein Tat induces apoptosis of hippocampal neurons by a mechanism involving caspase activation, calcium overload, and oxidative stress. Exp Neurol 1998; 154: 276-288.

23. Maragos WF, Young KL, Turchan JT, Guseva M, Pauly JR, Nath A et al. Human immunodeficiency virus-1 Tat protein and methamphetamine interact synergistically to impair striatal dopaminergic function. J Neurochem 2002; 83: 955-963.

24. Sahaf B, Atkuri K, Heydari K, Malipatlolla M, Rappaport J, Regulier E et al. Culturing of human peripheral blood cells reveals unsuspected lymphocyte responses relevant to HIV disease. Proc Natl Acad Sci USA 2008; 105: 5111-5116.
25. Kestler III HW, Ringler DJ, Mori K, Panicali DL, Sehgal PK, Daniel MD et al. Importance of the nef gene for maintenance of high virus loads and for development of AIDS. Cell 1991; 65: 651-662.

26. Xu W, Santini PA, Sullivan JS, He B, Shan M, Ball SC et al. HIV-1 evades virus-specific $\operatorname{lgG} 2$ and IgA responses by targeting systemic and intestinal $B$ cells via long-range intercellular conduits. Nat Immunol 2009; 10: 1008-1017.

27. Muratori C, Cavallin LE, Kratzel K, Tinari A, De Milito A, Fais S et al. Massive secretion by T cells is caused by HIV Nef in infected cells and by Nef transfer to bystander cells. Cell Host Microbe 2009; 6: 218-230.

28. Qiao X, He B, Chiu A, Knowles DM, Chadburn A, Cerutti A. Human immunodeficiency virus 1 Nef suppresses CD40-dependent immunoglobulin class switching in bystander B cells. Immunol 2006; 7: 302-310.

29. Lenassi M, Cagney G, Liao M, Vaupotic T, Bartholomeeusen K, Cheng Y et al. HIV Nef is secreted in exosomes and triggers apoptosis in bystander CD4+ T cells. Traffic 2010; 11: 110-122.

30. Ali SA, Huang MB, Campbell PE, Roth WW, Campbell T, Khan M et al. Genetic characterization of HIV type 1 Nef-induced vesicle secretion. AIDS Res Hum Retroviruses 2010; 26: 173-192.

31. Trillo-Pazos G, McFarlane-Abdulla E, Campbell IC, Pilkington GJ, Everall IP. Recombinant nef HIV-IIIB protein is toxic to human neurons in culture. Brain Res 2000; 864: 315-326.

32. Kramer-Hammerle S, Hahn A, Brack-Werner R, Werner T. Elucidating effects of long-term expression of HIV-1 Nef on astrocytes by microarray, promoter, and literature analyses. Gene 2005; 358: 31-38.

33. Acheampong E, Mukhtar M, Parveen Z, Ngoubilly N, Ahmad N, Patel C et al. Ethano strongly potentiates apoptosis induced by HIV-1 proteins in primary human brain microvascular endothelial cells. Virology 2002; 304: 222-234.

34. Acheampong EA, Roschel C, Mukhtar M, Srinivasan A, Rafi M, Pomerantz RJ et al. Combined effects of hyperglycemic conditions and HIV-1 Nef: a potential model for induced HIV neuropathogenesis. Virol J 2009; 6: 183

35. Studer L, Csete M, Lee SH, Kabbani N, Walikonis J, Wold B et al. Enhanced proliferation, survival, and dopaminergic differentiation of CNS precursors in lowered oxygen. $J$ Neurosci 2000; 20: 7377-7383.

36. Reichert SA, Kim-Han JS, Dugan LL. The mitochondrial permeability transition pore and nitric oxide synthase mediate early mitochondrial depolarization in astrocytes during oxygen-glucose deprivation. J Neurosci 2001; 21: 6608-6616.

37. Abramov AY, Scorziello A, Duchen MR. Three distinct mechanisms generate oxygen free radicals in neurons and contribute to cell death during anoxia and reoxygenation. J Neurosci 2007; 27: 1129-1138.

38. Azad AA. Could Nef and Vpr proteins contribute to disease progression by promoting depletion of bystander cells and prolonged survival of HIV-infected cells? Biochem Biophys Res Commun 2000; 267: 677-685.

Cell Death and Disease is an open-access journal published by Nature Publishing Group. This work is licensed under the Creative Commons Attribution-Noncommercial-Share Alike 3.0 Unported License. To view a copy of this license, visit http:// creativecommons.org/licenses/by-nc-sa/3.0/

\section{Supplementary Information accompanies the paper on Cell Death and Disease website (http://www.nature.com/cddis)}

